\title{
LE SALAIRE MINIMUM INTERPROFESSIONNEL GARANTI SOUS LA CONSTITUTION DU 18 FEVRIER 2006. CADRE JURIDIQUE ET PROBLEMES D'APPLICATION EFFECTIVE EN RD CONGO
}

\author{
Par NSAMBANA BONKAKO Juslain*
}

\section{Résumé}

Le décret $n^{\circ} 18 / 017$ du 22 mai 2018 portant fixation du nouveau SMIG a fixé celui-ci à 7.075CDF, représentant 5USD au moment de la prise de décision. Ce décret, qui aurait pour vocation de remplacer, en l'abrogeant (sic), l'ordonnance $n^{\circ} 80 / 040$ du 30 avril 2008 ayant fixé le SMIG à 1.680CDF, tire son fondement légal de l'article 87 du code du travail congolais de 2002 qui reconnait cette compétence au Président de la République.

Dans cette étude, la réflexion est orientée à trois niveaux : il est d'abord question de ressortir, à la lumière de la lecture des articles 87 du code du travail de 2002 et 204, point 17 de la constitution de la RDC du 18 février 2006, l'autorité compétente en matière de fixation du SMIG, ce qui permet de tirer les conséquences du décret $n^{\circ} 18 / 017$ du 22 mai 2018 , précité ; ensuite, présenter le champ d'application du SMIG où il importe de préciser notamment entre rémunération et salaire, lequel sert d'élément de comparaison au SMIG, quels types et catégories de salariés sont-ils concernés par son application ; enfin, l'approche empirique consistant à aborder des problèmes pratiques que suscite l'application du SMIG entre employeurs et travailleurs.

\begin{abstract}
The Decree $n^{\circ} 18 / 017$ of 22 May 2018 setting the new minimum SMIG fixed it at 7,075CDF, representing 5USD at the time of the decision. This decree, which is intended to replace, by repealing (sic), the Order No. 80/040 of 30 April 2008, which set the SMIG at 1,680 CDF, derives its legal basis from Article 87 of the 2002 Congolese Labour Code, which recognises the President of the Republic's authority to set the SMIG.

In this study, the reflection is oriented on three levels: First, it is a question of identifying, in the light of the reading of articles 87 of the 2002 Labour Code and 204, point 17 of the Constitution of the DRC of 18 February 2006, the competent authority for setting the minimum SMIG, which makes it possible to draw the consequences of the Decree No. 18/017 of 22 May 2018, cited above; secondly, to present the scope of application of the $S M I G$, in which it is important to specify, in particular, between remuneration and salary,

* Assistant du Professeur Jean-Michel KUMBU de la Faculté de droit, Université de Kinshasa ; Apprenant au III ${ }^{\mathrm{ème}}$ Cycle - Unikin, Chercheur au CREEDA (RDC); jnsambana@gmail.com.
\end{abstract}


which serves as an element of comparison with the SMIG, what types and categories of employees are concerned by its application; finally, the empirical approach consisting in addressing practical problems arising from the application of the SMIG between employers and workers.

\section{Introduction}

L'homme depuis jadis est préoccupé par l'amélioration de ses conditions de vie. La recherche du bien-être hante chaque jour l'homme et finit par l'épuiser. Cet épuisement peut se justifier par l'incapacité de l'homme à subvenir à ses besoins par ses propres économies. Dans une société hautement stratifiée et spécialement capitaliste, l'homme est au service de l'homme pour survivre. Cette relation de subordination renforce l'autorité du patronat tout en déséquilibrant les rapports en présence.

Le travail est à la fois un droit et un devoir sacrés pour chaque Congolais ${ }^{1}$. Ce principe constitutionnel fait ressortir deux réalités apparemment opposées mais dont la coexistence se justifie véritablement par, d'abord l'obligation pour l'Etat d'assurer l'emploi à toute personne dans la mesure où le bien-être en dépend, ensuite, l'obligation pour chacun de participer, par son travail, au développement de l'Etat en ce qu'il est démontré que le travail est une des sources de développement ${ }^{2}$.

Au droit de travailler, s'attache celui de rémunérer la personne qui fournit ses prestations au profit de l'autre. La rémunération est définie comme la somme représentative de l'ensemble des gains susceptibles d'être évalués en espèces et fixés par un accord ou par des dispositions légales ou réglementaires qui sont dus en vertu d'un contrat de travail, par un employeur à un travailleur ${ }^{3}$. C'est de façon élémentaire le prix du travail fourni par une personne physique au profit d'une autre personne, physique ou morale, en vertu d'un contrat de travail.

Il a longtemps été présenté le risque du déséquilibre des forces dans les relations professionnelles entre employeurs et travailleurs, surtout dans le monde capitaliste où priment les intérêts du patron au détriment de ceux des ouvriers. Face à la nécessité d'assouplir ce dés-

1 Article 36, alinéa ${ }^{\text {er }}$ de la constitution de la RDC du 18 février 2006, telle que révisée par la loi n •11/002 du 20 janvier 2011 portant révision des certains articles de la constitution.

2 Le professeur Jean-Michel KUMBU ki NGIMBI présente en effet un schéma qui part du travail au développement, en passant par l'accroissement des richesse qui en est la conséquence et la bonne gouvernance, le lire dans son manuel de Droit social, Kinshasa, 2018, p.

3 Article 7, point 8 de la loi $n^{\circ} 015-2002$ du 16 octobre 2002 portant code du travail, telle que modifiée par la loi $\mathrm{n}^{\circ} 16 / 10$ du 15 juillet 2016. Cette définition du législateur congolais écarte certains autres revenus obtenus par le travailleur à l'occasion de son contrat de travail. C'est en ce sens qu'il n'est pas sans intérêt de reprendre également la définition de G.H CAMERLYNCK qui considère la rémunération comme " toute somme ou tout avantage accordé à l'occasion du travail dans le cadre de l'entreprise ayant pris le travailleur à charge " (G.H CARMELYNCK et LYON-CAEN, Droit du travail, Paris, 1965, p.179). 
équilibre ${ }^{4}$, l'obligation pour les pouvoirs publics d'intervenir, en tant que régulateur, non seulement par la production des normes juridiques mais aussi en instituant des organes de contrôle ayant la mission de veiller au respect des prescriptions légales, s'est justifiée.

Parmi ces normes instituées, citons notamment l'arsenal juridique qui forme le droit du travail dont les sources sont à la fois internes et internationales, lesquelles accordent une place importante à la rémunération, l'un des éléments caractéristiques du contrat de travail.

La rémunération contient divers éléments parmi lesquels le plus déterminant est évidemment le salaire. Celui-ci est généralement fixé par l'employeur qui, au moment de l'embauche, le propose comme clause contractuelle. Dans la plupart des cas, le travailleur l'accepte et dès lors, le contrat de travail est formé, le consentement des parties sur les différentes clauses contractuelles, y compris sur le salaire, étant obtenu. C'est donc généralement un contrat d'adhésion qui, dans les pays à taux de chômage élevé, le travailleur n'a pas assez de marges de négociations. En pareil cas, l'employeur souvent capitaliste n'a aucun intérêt à proposer un salaire décent au travailleur. Au contraire, ce qui le préoccupe c'est la réduction des charges du personnel en vue d'accorder assez de chance à ses profits. La tendance des entreprises à se délocaliser pour s'installer dans des pays dont la main d'œuvre ne coûte pas assez justifie cette thèse.

En vue de prévenir les abus des employeurs dans la détermination du salaire, des gardefous ont été sensiblement développés tant au niveau des pouvoirs publics que des organisations ouvrières, appelées syndicats, parmi lesquels relevons en ce qui nous concerne la fixation du salaire minimum interprofessionnel garanti (SMIG). Ces garde-fous se présentent ainsi comme des principes d'ordre public institués dans le monde privé en vue de restreindre la liberté de l'employeur et préserver les droits des travailleurs, considérés comme économiquement faibles.

Le SMIG (appelé sous d'autres cieux Salaire Minimum Interprofessionnel de Croissance, SMIC) est le salaire journalier ou horaire, selon les pays, fixé par l'autorité compétente en-dessous duquel aucun salarié ne doit en principe être payé. Il a pour objet de garantir aux salariés dont les rémunérations sont les plus faibles le maintien de leur pouvoir d'achat ainsi qu'une participation au développement économique de la nation ${ }^{5}$.

Les analyses économiques de l'employeur suscitent cependant certaines interrogations en rapport avec le principe posé sur l'application du SMIG en vue de garantir le minimum salarial à tous les travailleurs. Il est en effet une catégorie d'employeur dont le chiffre d'affaires, si minime qu'il est, n'atteint parfois pas le montant permettant de résorber le minimum salarial fixé.

4 Il est difficile d'arriver à l'équilibre entre travailleur et employeur, surtout dans des pays où obtenir l'emploi relève du domaine de la foi, mieux, des miracles, obligeant à tort ou à raison ceux qui le cherchent à pratiquer n'importe quelle expérience, sous la conduite des « serviteurs de Dieu ».

5 Franck BAVOZET, Social 2016, Paris, 2016, p. 861. 
Trois questions méritent une réflexion dans le cadre de cette étude :

- D'abord, qu'en est-il de la compétence de fixation du salaire minimum interprofessionnel garanti en RD Congo ?

- Sur quels salariés s'applique-t-il ?

- Quels sont les problèmes pratiques que suscite l'application du salaire minimum interprofessionnel garanti?

Ces interrogations énoncent les différents points à examiner dans les lignes qui suivent.

\section{A. Compétence en matière de fixation des salaires minima interprofessionnels garantis}

L'Etat est constitué de plusieurs organes exerçant les pouvoirs, selon une certaine répartition contenue soit dans la constitution, soit dans les différents textes législatifs. C'est dans cette logique que l'article 204 de la constitution de la $\mathrm{RDC}^{6}$ dispose : sans préjudice des autres dispositions de la présente constitution, les matières suivantes sont de la compétence exclusive des provinces :...17 la fixation des salaires minima provinciaux, conformément à la législation nationale... ».

Le régionalisme politique consacré comme forme de l'Etat en RD Congo par la constitution du 18 février $2006^{7}$, telle que révisée en 2011, suscite beaucoup de réflexions, non seulement en droit constitutionnel, mais également en finances publiques et, en ce qui nous concerne, en droit du travail. Le point 17 de l'article 204, précité, est à l'origine de la problématique qui consiste à s'interroger à la fois sur l'avenir de la zone unique du SMIG institué en RD Congo par le législateur social et sur l'autorité compétente pour décider des salaires minima interprofessionnels garantis, depuis la promulgation de la constitution actuelle.

\section{Compétence en matière de fixation des salaires minima avant la constitution du 18 février 2006}

Avant l'actuelle constitution (de 2006, telle que révisée), le salaire minimum interprofessionnel garanti avait comme base juridique, le code du travail du 16 octobre 2002, aux articles 87 à 92.

6 Constitution du 18 février 2006 telle que modifiée par la loi n $11 / 002$ du 20 janvier 2011 portant révision des certains articles de la constitution du 18 février 2006.

7 Sans se verser dans ce débat autour de la forme de l'Etat consacrée par la constitution du 18 février 2006, que nous renvoyons à d'autres, ce qui est préoccupant dans cette étude, c'est la question de répartition des compétences entre le pouvoir central et les provinces, spécialement sur les compétences exclusives qui ne peuvent pas, et donc pas du tout, susciter des questions de conflit de compétence ni de chevauchement. Lire au sujet de la forme de l'Etat : A. MAZYAMBOMAKENGO KI$S A S A$, Régionalisme et décentralisation en République Démocratique du Congo, in La consolidation du cadre démocratique en République démocratique du Congo, modules de renforcement des capacités à l'intention des institutions parlementaires, PNUD, Kinshasa, pp. 27-33. 
L'article 87 du code du travail dispose en effet qu'un décret du président de la République, pris sur proposition du Ministre ayant le Travail et la Prévoyance sociale dans ses attributions, après avis du Conseil National du Travail, fixe les salaires minima interprofessionnels garantis ainsi que les taux des allocations familiales minima, et à défaut de conventions collectives ou dans le silence, les salaires minima par catégorie professionnelle.

La fixation du SMIG, étant du domaine réglementaire, relève de la compétence du Président de la République qui agit sur proposition du Ministre ayant le Travail et la Prévoyance sociale dans ses attributions, malgré que l'article précité évoque le décret. La justification est simple en ce que le code du travail, promulgué et publié en 2002, devait se conformer à la constitution en vigueur à cette date, à savoir le décret-loi constitutionnel de 1997 pris par le président Laurent Désiré KABILA, qui prévoyait que le Président de la République agissait par voie de décret ${ }^{8}$. C'est donc à tort de considérer qu'en harmonie avec cette disposition, le Premier Ministre serait compétent en la matière. La nature de la décision, telle que reprise dans le code du travail n'est susceptible d'aucune confusion, le premier ministre n'est pas compétent pour fixer le SMIG.

\section{Compétence en matière de fixation des salaires minima depuis la constitution du 18 février 2006}

La constitution du 18 février 2006, telle que révisée en 2011, reprend la fixation des salaires minima provinciaux parmi les matières relevant de la compétence exclusive des provinces. C'est dire que s'agissant de la réflexion au sujet de la recherche de l'organe compétent dans la fixation des salaires minima, l'on peut, avant toute analyse approfondie, être tenté d'écarter les organes du pouvoir central. Le débat se situerait entre l'exécutif provincial et son législatif. Ainsi, dans la mesure où la fixation des salaires minima relève du domaine réglementaire, il appartiendrait au Gouverneur de province de les fixer, par voie d'arrêté du gouverneur.

Cependant, la question est de savoir si, en parlant des salaires minima provinciaux, le constituant de 2006 faisait allusion aux salaires minima interprofessionnels garantis prévus par le législateur social qui consacre en même temps la zone unique du $\mathrm{SMIG}^{9}$, auquel cas la réflexion faite ci-haut tiendrait pleinement. Mais dans le cas contraire, y aurait-il des salaires minima interprofessionnels garantis fixés par le Président de la République conformément au code du travail qui ont vocation à s'appliquer sur toute l'étendue du territoire national, parallèlement à d'autres salaires minima provinciaux qui, eux, s'observent sur l'étendue de la province concernée?

Les salaires minima provinciaux renverraient à l'idée des salaires à fixer par la province à l'égard des salariés ne relevant ni du secteur privé, ni de pouvoir central dont le barème salarial tient compte du budget général de l'Etat tel que soumis et voté au Parlement. Juridi-

8 Article 5 du décret-loi constitutionnel de 1997 (RDC).

9 Article 91 du code du travail. 
quement, la province, en tant que personne morale de droit public, peut valablement « employer » des agents dont le salaire dépend directement d'elle, conformément à son budget, et non du pouvoir central. C'est en ce sens, à notre avis, que la constitution évoque la fixation des salaires minima provinciaux comme matière réservée exclusivement aux provinces.

Il importe de soutenir qu'agissant, en vertu de la constitution du 18 février 2006, par voie d'ordonnance, le salaire minimum interprofessionnel garanti tel que prévu dans le code du travail doit être fixé par une ordonnance du Président de la République, prise sur proposition du Ministre ayant le Travail et la prévoyance sociale dans ses attribution, après que ce dernier ait requis l'avis du Conseil National du Travail.

\section{Analyse de quelques textes fixant le SMIG sous la constitution du 18 février 2006}

Depuis la promulgation de l'actuelle constitution, deux textes ont été pris pour fixer le SMIG, à savoir : l'ordonnance 80/040 du 30 avril 2008 et le décret $n^{\circ}$ 18/017 du 22 mai 2018.

1. De l'ordonnance $n^{\circ} 80 / 040$ du 30 avril 2008 portant fixation du salaire minimum interprofessionnel garanti, des allocations familiales minima et de la contre-valeur du logement

Contenant quatorze articles, l'ordonnance n ${ }^{\circ} 80 / 040$ du 30 avril 2008 portant fixation du salaire minimum interprofessionnel garanti, des allocations familiales minima et de la contrevaleur du logement a été prise pour remplacer le décret $n^{\circ} 80 / 2002$ du 3 juillet 2002 portant fixation du salaire minimum interprofessionnel garanti, des allocations familiales minima et de la contre-valeur du logement, en vue de promouvoir la solidarité nationale, la réhabilitation de la culture du travail décent, la productivité et la sécurité des affaires ${ }^{10}$.

Cette ordonnance fixait le taux journalier du salaire minimum interprofessionnel garanti à $1.680 \mathrm{CDF}$ pour le manœuvre ordinaire ${ }^{11}$. Ce montant était appliqué en deux paliers dont le premier, égal à $1.120 \mathrm{CDF}$, payable à partir du $1^{\mathrm{er}}$ juillet 2008 et la totalité, soit 1.680CDF, à partir du $1^{\mathrm{er}}$ janvier $2009^{12}$; en respectant la tension salariale de 1 à 10 ou de 100 à $1000^{13}$, laquelle part du manœuvre ordinaire au cadre de collaboration.

Le nouveau SMIG, aussi dérisoirement évalué à $3 \mathrm{USD}$, a été pourtant salué par la plupart des travailleurs ${ }^{14}$, quoiqu'il convient de relever qu'au moment de la décision, certains salaires respectaient déjà ce minimum avant même qu'il soit décidé.

10 Ceci ressort de l'exposé de motif de l'ordonnance précitée.

11 Article 2 de l'ordonnance $n^{\circ} 80 / 040$ précitée.

12 Article 3 de la même ordonnance.

13 Article 4 de la même ordonnance.

14 Salué, pas parce qu'ils ont trouvé satisfaction au nouveau SMIG au regard des fonctions que le salaire est appelé à remplir dans la vie sociale du travailleur, mais en comparaison à celui qu'il remplace. 
Le problème du SMIG en RDC est plutôt lié au tissu économique du pays. En effet, le SMIG étant toujours fixé en monnaie ayant cour légal au Congo, à savoir le franc congolais, se dévalue en même temps que la dévaluation de l'unité monétaire congolaise. Le SMIG de $1.680 \mathrm{Fc}$ tant applaudi en son temps a, au fil de temps, perdu toute sa valeur pour représenter un dollars américain au lieu de trois, du fait de la dévaluation monétaire dont les causes sont à la fois économiques, politiques que juridiques. C'est cela qui est à la base du dynamisme du SMIG. Le SMIG devra dans un tel contexte être revu toutes les fois que les données économiques en présence ne concordent plus avec le montant fixé.

Comme celui qu'il a remplacé, le SMIG de 2008 est lui aussi devenu modique pour les travailleurs ; son remplacement devenait donc imminent. C'est en ce sens que la procédure de détermination d'un nouveau SMIG a été déclenchée en 2018.

\section{Du décret $n^{\circ} 18 / 017$ du 22 mai 2018 portant fixation du nouveau SMIG}

Le SMIG tel que fixé par l'ordonnance précitée a sensiblement été terrassé par les données économiques actuelles; de sorte qu'il est devenu un monstre pour les travailleurs alors que sa fixation a pour objectif d'assurer leur bien-être social, en garantissant leur pouvoir d'achat. Fixé à $1.680 \mathrm{CDF}$, l'équivalent de 3 dollars américains au moment de la prise de décision, ce SMIG est, dix ans après, devenu plus en plus dénigrant pour les travailleurs en ce qu'au regard du taux de change actuel, il ne représente qu'un dollars américain par jour pour un travailleur manœuvre ordinaire. En outre, son réajustement au taux actuel n'aurait pas non plus réglé le problème ; il était impérieux d'entrevoir un nouveau SMIG en remplacement de celui devenu obsolète.

Ainsi, à l'occasion de la $33^{\text {ème }}$ session ordinaire du Conseil National du Travail, les partenaires concernés, à savoir la représentation des employeurs, celle des travailleurs et la partie gouvernementale, s'étaient évidemment rendus compte de cette réalité. Les échanges ont abouti à la conclusion de la révision du SMIG susceptible de garantir aux travailleurs le minimum vital. Au terme desdits échanges, un avis a été émis au ministre ayant le travail et la prévoyance sociale dans ses attributions aux fins de proposer à l'autorité compétente la fixation du SMIG.

Tout juriste préoccupé par le domaine du travail s'attendait à une ordonnance fixant le SMIG, laquelle aurait le mérite d'abroger l'ordonnance n80/040 précitée, il a plutôt été question d'un décret (acte du premier ministre) qui sera publié au journal officiel en date du 11 juin 2018. Pendant que des réactions de joie se manifestaient pour la plupart, se battant ainsi à obtenir son respect par les employeurs et à prévoir les modalités de son application ${ }^{15}$, juridiquement des questions se posent notamment sur les effets d'une décision prise

15 Il y a lieu de reconnaitre les efforts fournis par le Département Juridique, Social et fiscal de la Fédération des Entreprises du Congo (FEC) dans la mise en œuvre explicative du SMIG tel que fixé par ce fameux décret n 18/017 du 22 mai 2018. (Lire FEC, Département Juridique, Social et Fiscal, « le salaire minimum interprofessionnel garanti en application en République Démocratique du Congo », in Veillé juridique en entreprise, n007/DJSF/2018, novembre 2018, pp 1-3. 
par une autorité qui n'en est pas compétente ainsi que sur sa conséquence par rapport à l'ordonnance qu'il ne peut pas abroger, n'étant pas un acte de la même nature.

Il semble qu'avant la prise de décision fixant le SMIG, les employeurs, à travers leur représentant (la FEC), avait fait observer la portée de l'article 204, point 17 de la constitution que reconnait aux provinces la compétence exclusive de fixer les salaires minima provinciaux et qu'une suggestion avait été faite d'adopter que « le taux journalier du SMIG tel qu'adopté, à savoir $7.075 \mathrm{FC}$, constitue le maximum du taux journalier des salaires minima provinciaux que les provinces devront scrupuleusement respecter lors de la fixation des taux spécifiques propres à chacune d'elles ». La suggestion n'a cependant pas été retenue, le seul taux du SMIG a été maintenu sans faire allusion à la disposition constitutionnelle ${ }^{16}$.

L'on peut ainsi retenir que l'actuel SMIG fixé par le décret (du premier ministre) n'emporte pas notre conviction compte tenu de l'incompétence de l'autorité ayant pris la décision. En effet, de toutes les deux hypothèses, ci-haut présentées, tirées de la lecture de l'article 87 du code du travail et 204, point 17 de la constitution de la RDC, le premier ministre n'a aucune compétence en matière de fixation du SMIG. De cette logique, le décret sous examen ayant fixé le SMIG journalier à 7.075CDF (équivalent de 5USD au moment de la décision) ne peut juridiquement pas abroger et remplacer les dispositions de l'ordonnance 80/040 du 30 avril 2008, précitée.

\section{B. Champs d'application du salaire minimum interprofessionnel garanti}

Ce point est à examiner à trois niveaux : d'abord au plan matériel (sur quel élément de rémunération et sa conséquence sur d'autres), ensuite préciser sur quelle catégorie des salariés (privés et publics ou privés uniquement ? nationaux uniquement ou les expatriés aussi ?) et enfin, le contrôle de l'application du SMIG : compétence et réalité pratique.

\section{Le salaire minimum interprofessionnel garanti : sur quoi opère-t-il entre rémunération et salaire?}

\section{Tentative d'identification d'élément de comparaison du SMIG}

Ce point concerne des travailleurs dont le revenu mensuel est constitué de plusieurs éléments formant la structure salariale. Il est donc sans intérêt à l'égard de ceux dont le revenu est globalement déterminé, comme c'est généralement le cas dans les petites et moyennes entreprises.

La notion de rémunération et celle de salaire suscitent déjà des interrogations non seulement dans la législation congolaise mais également ailleurs. Contrairement au salaire que le législateur congolais ne définit pas, aux termes de l'article 7, point 8 du code du travail, la rémunération est la somme représentative de l'ensemble des gains susceptibles d'être éva- 
lués en espèces et fixés par accord ou par les dispositions légales et réglementaires qui sont dus en vertu d'un contrat de travail, par un employeur à un travailleur.

Au sujet de la rémunération, la même disposition énumère les éléments qui la forment. Il s'agit notamment du salaire ou traitement, des commissions, de l'indemnité de vie chère, des primes, de la participation aux bénéfices, des sommes versées à titre de gratification ou de mois complémentaire (appelé généralement treizième mois), des sommes versées pour prestations supplémentaires, la valeur des avantages en nature, l'allocation de congé ou l'indemnité compensatoire de congé, les sommes payées par l'employeur pendant l'incapacité de travail et pendant la période précédant et suivant l'accouchement. A côté de ces éléments de rémunération, il existe d'autres ressources qui apparaissent dans la paie des travailleurs que le législateur ne range pas comme éléments de rémunération. Il s'agit limitativement des soins de santé, de l'indemnité de logement ou le logement en nature, les allocations familiales légales, l'indemnité de transport, les frais de voyage ainsi que les avantages accordés exclusivement en vue de faciliter au travailleur l'accomplissement de ses fonctions.

En réalité tous ces éléments constituent le revenu mensuel du travailleur ${ }^{17}$; leur distinction par le législateur se fonde surtout au niveau des conséquences qu'ils entrainent, en ce que ceux qui figurent au premier groupe concourent à la constitution de l'assiette imposable à l'IPR, à la cotisation de sécurité sociale, etc. ; alors que la seconde catégorie est à écarter pour la simple raison de leur caractère de remboursement. En effet, pour ces derniers éléments, il revient en principe à l'employeur de prendre en charge les différents services couverts par ces paiements. C'est le cas du transport dont l'obligation revient à l'employeur de mettre à disposition des travailleurs un moyen de locomotion permettant leur déplacement, allé et retour, de leur résidence au lieu de travail. Faute de pouvoir le faire, une indemnité est payée à ceux-ci, qui se comportent en réalité comme intermédiaire, afin de le reverser au transporteur. Le même raisonnement tient en ce qui concerne le logement et les soins de santé.

De tous ces éléments constitutifs de l'enveloppe salariale du travailleur, le salaire demeure incontestablement la base qui ne peut en aucun cas ne pas figurer. Contrairement à ces autres éléments fragiles dont la détermination est parfois laissée à l'appréciation de l'employeur, le salaire répond à une autre logique de détermination.

La question de déterminer, entre rémunération et salaire lequel doit ne pas être inférieur au SMIG suivant le principe posé par le législateur du code du travail, est embarrassante. L'embarra provient du législateur qui utilise parfois l'un pour l'autre. La majorité des doc-

17 Ce revenu est en réalité qualifié de rémunération par la convention $n^{\circ} 100$ de l'OIT ratifiée par la $\mathrm{RDC}$, laquelle n'est pas dans la logique du législateur congolais qui exclut certains éléments de la rémunération. Selon cette convention, la rémunération comprend le salaire ou traitement ordinaire, de base ou minimum, et tous autres avantages, payés directement ou indirectement, en espèce ou en nature, par l'employeur au travailleur en raison de l'emploi de ce dernier. (OIT, Conventions et recommandations internationales du travail 1914-1984, volume I, BIT, Genève, 1985, p. 45). 
trinaires reconnaissent en effet que le salaire est un élément de la rémunération ${ }^{18}$. L'affirmation du professeur DIUMI SHUTSHA ${ }^{19}$ qui relève, raisonnablement, qu'il est parfois difficile de suivre le législateur social de manière soutenue lorsqu'il traite de la question de salaire, mérite toute notre approbation. L'article 7, point 8 précité, reprend le salaire comme élément de la rémunération; il est dès lors simple d'affirmer avec le professeur KUMBU ki $\mathrm{NGIMBI}^{20}$, que la rémunération est un grand ensemble dans lequel on y trouve notamment le salaire. Si les termes de "rémunération » et de "salaire » sont, dans la plupart des cas, employés dans le même sens, la notion de rémunération peut présenter un caractère plus global et viser toutes les sommes versées au salarié ${ }^{21}$. De ce fait, il est invraisemblable que dans un chapitre qui traite de salaire, le législateur parle de la rémunération. Est-ce une confusion qui serait analysée en une erreur du législateur et rester dans l'esprit qui consisterait à attribuer ce qui est dit au salaire, malgré que littéralement la loi utilise la rémunération?

La confusion du législateur est la conséquence de ne s'être pas conformé à la convention $\mathrm{n}^{\circ} 100$. Cette dernière a l'avantage de préciser, dans l'énumération des éléments de la rémunération, l'élément salaire ou traitement ordinaire, pouvant être de base ou minimum, différent des autres avantages qui l'accompagnent pour constituer la rémunération du travailleur.

Suivant cette logique cohérente de la convention n¹00, il est sans ambigüité de soutenir que le SMIG se compare au salaire ou traitement de base, entendu comme élément de la rémunération et non à la rémunération dans son ensemble ou, même dans la logique confuse du législateur congolais, au cumul du salaire et des autres éléments qui suivent son régime.

\section{Les différents paramètres d'application du SMIG}

Le salaire minimum interprofessionnel garanti tient compte de la tension salariale qui s'examine en tenant compte de la catégorisation professionnelle au sein d'une entreprise. Selon l'option prise, cette tension va de 1 à 10 ou de 100 à 1000 . Chaque tension correspond à une catégorie professionnelle précise.

L'employeur a dès lors l'obligation d'appliquer une classification contenant tous les emplois d'exécution, de maitrise jusqu'au cadre de collaboration. L'emploi de cadre de collaboration vise le travail exercé par un salarié qui ne possède pas le pouvoir de décider en toute autonomie de manière à influencer fondamentalement la bonne marche de l'entreprise $^{22}$.

18 Notamment MASANGA PHOBA MVIOKI, Droit du travail, Kinshasa, 2018.

19 D. DIUMI SHUTSHA, Six leçons de droit du travail, Kinshasa-Bruxelles, 2018, p. 426.

$20 J M$ KUMBU ki NGIMBI, Droit social, manuel d'enseignement, Kinshasa, 2018, pp. 1-5.

21 Franck BAVOZET, note 5, p. 850.

22 D. DIUMI SHUTSHA, note 8, p. 426. 
Dans la détermination du SMIG, le taux journalier qui est fixé correspond à la dernière catégorie professionnelle ayant la tension 1 ou 100, selon le cas, à savoir le travailleur manœuvre ordinaire. De ce fait, chaque employeur, en fonction de la classification professionnelle applicable au sein de l'entreprise, a l'obligation d'arrêter le salaire de chaque catégorie professionnelle en se basant sur la tension salariale correspondante. En 2008 par exemple, le taux journalier fixé à 1680CDF (l'équivalent à l'époque de 3 dollars américain) correspondait au SMIG journalier du manœuvre ordinaire. Ainsi, pour déterminer le SMIG du cadre de collaboration, ayant la tension 8 , il est donc question de multiplier 1680 par 8 .

\section{Personnes concernées par le salaire minimum interprofessionnel garanti}

Dans chaque pays, il existe diverses catégories de salariés, selon la nature des employeurs. Certains sont salariés de l'Etat, appelés fonctionnaires sous leurs diverses qualifications ; d'autres sont employés des organismes internationaux et ambassades ; et enfin, la dernière grande catégorie appartenant au secteur privé. En conformité aux règles établies, toutes ces différentes catégories professionnelles font participer les nationaux et les expatriés, la discrimination à l'emploi pour raison de nationalité étant formellement prohibée ${ }^{23}$.

1. Application du SMIG : qui de salariés privés et publics sont soumis au SMIG

La recherche des salariés soumis au SMIG renvoie à la question d'identifier lesquels des salariés sont soumis au code du travail. Du point de vue de son objectif qui consiste à assurer le bien-être aux salariés en luttant pour que le minimum de salaire ainsi fixé soit à même de protéger le pouvoir d'achat du bénéficiaire, l'on peut être tenté de considérer que le SMIG serait imposable à tous les employeurs, privés et publics, y compris l'Etat - premier et plus grand employeur.

Néanmoins, en recherchant les fondements juridique et philosophique du SMIG, l'on peut aisément soutenir que seuls sont concernés les salariés dont les rapports professionnels avec employeurs sont régis par la législation sociale, à savoir le code du travail et le texte sur la sécurité sociale ${ }^{24}$, complétés par les différents textes réglementaires.

Le SMIG, rappelons-le, constitue l'un des mécanismes d'interventionnisme étatique dans la détermination du salaire qui, par essence, est une question relevant de l'échange des volontés entre employeur et travailleur, soit dans le contrat, soit dans la convention collective. Le salaire, élément essentiel du contrat de travail, est très déterminant dans la validité

23 Notamment la convention n ${ }^{\circ} 00$ sur l'égalité de rémunération du 29/06/1951; la Recommandation n 90 sur l'égalité de rémunération du 29/06/1951; la Convention n 111 concernant la discrimination (emploi et profession) du 25/06/1958 ; la Recommandation $n^{\circ} 111$ concernant la discrimination (emploi et profession) du 25/06/1958 ; la Constitution de la RDC du 18 février 2006 telle que modifiée par la loi n ${ }^{\circ} 11 / 002$ du 20 janvier 2011 portant révision des certains articles de la constitution du 18 février 2006 ; la loi $\mathrm{n}^{\circ}$.

24 Spécialement la loi nº $16 / 009$ du 15 juillet 2016 fixant les règles relatives au régime général de sécurité sociale qui concerne principalement le secteur privé. 
même du contrat de travail en ce qu'en tant que contrat synallagmatique qui requiert la réciprocité des obligations, le consentement du travailleur, dans un contexte où il a la liberté spirituelle ${ }^{25}$ d'appréciation, est le plus souvent tributaire de deux importantes conditions, en l'occurrence la nature des prestations et le prix desdites prestations qui n'est rien d'autre que le salaire.

Idéologiquement, par le SMIG, l'Etat intervient pour constituer un contrepoids à l'employeur, dans l'intérêt des travailleurs. En ce sens, en tant qu'employeur, il est absurde de soutenir qu'il (l'Etat) prenne des mesures fixant les bornes de ses propres actions. Le SMIG est donc une affaire des salariés et employeurs du secteur privé et de public-privé ${ }^{6}$. Logiquement, le barème salarial que fixe l'Etat en faveur de ses salariés ne devait pas être inférieur au SMIG applicable, pas parce qu'il y est soumis mais pour justifier « la charité bien ordonnée ».

La seconde justification, de nature juridique, est celle qui s'appuie sur le fondement juridique du SMIG. En effet, sans remettre en cause les analyses faites au point I sur la compétence en matière de détermination du SMIG, ayant posé le principe à l'article 86 selon lequel à conditions égales de travail, de qualification professionnelle et de rendement, le salaire est égal pour tous les travailleurs, quels que soient leur origine, leur sexe et leur age, le législateur du code du travail impose aux pouvoirs publics l'obligation de fixer par voie réglementaire les salaires minima interprofessionnels garantis ainsi que les taux des allocations familiales minima ${ }^{27}$, en prescrivant de nullité ${ }^{28}$ toute clause de contrat individuel ou de convention collective fixant des rémunérations (salaires normalement) inférieures auxdits salaires minima ${ }^{29}$.

25 Dans un monde de carence d'emploi, le candidat à l'embauche est, certes, libre d'accepter ou de refuser un emploi dans la mesure où le travail forcé est formellement interdit ; cependant, cette liberté est difficile à faire valoir si l'on sait qu'aucune autre offre n'est disponible. Il arrive d'ailleurs que pour autant de postes publiés dans les rares offres d'emploi, le nombre des candidats devienne ingérable. Le candidat se voit dès lors contraint de donner son consentement, même sans qu'il ait eu préalablement connaissance du salaire déterminé. La liberté d'esprit se trouve alors sensiblement entamée.

26 Par public-privé, nous faisons allusion aux établissements publics qui emploient des travailleurs régis pas le code du travail ; sans faire la redondance en citant ceux des sociétés commerciales issues de la transformation des entreprises publiques, qui sont déjà soumises au droit commun des sociétés commerciales (privées). C'est le cas par exemple de l'INPP et son personnel, de la CNSS, de l'OGEFREM, de l'OCC, etc.

27 Article 87 du code du travail de 2002.

28 Le point III de cette étude aborde la question de la nature de cette nullité en examinant la possibilité ou non pour les parties au contrat du travail de se compromettre et de convenir à un salaire inférieur au SMIG.

29 Il n'y a pas de nullité sans texte ; en effet l'article 88, alinéa 2 du code du travail dispose qu' « est nulle de plein droit toute clause de contrat individuel ou de convention collective fixant des rémunérations inférieures aux salaires minima interprofessionnels garantis déterminés conformément à l'article $87 »$. 
Il est aisé à affirmer qu'au vu de ces dispositions légales sur le salaire, les règles fixant le SMIG sont opposables aux personnes dont les rapports sont régis par le code du travail, à savoir les travailleurs et employeurs du secteur privé et ceux du secteur public-privé.

L'exclusion des salariés du secteur public, à savoir les fonctionnaires et autres agents de l'Etat, se justifie dans la dépendance de la détermination de leur salaire au budget de l'Etat dont les mécanismes d'élaboration, de vote et de contrôle s'éloignent véritablement de la logique contractuelle du secteur privé. Dans le budget de l'Etat, les lignes budgétaires en termes des charges sont arrêtées en tenant compte de la capacité de mobilisation des recettes.

En outre, la logique de catégorisation professionnelle opérationnelle dans le secteur privé ne correspond pas à l'organisation des grades au sein de l'administration publique dans sa diversité.

Dans tous les cas, quoique non soumis au SMIG, l'Etat devait fixer le salaire de ses salariés en tenant compte d'un barème susceptible de ne pas tomber en deçà du minimum légal fixé pour les autres.

Concernant les seuls salariés soumis au SMIG, l'article 90 du code du travail prévoit que l'employeur est tenu d'appliquer une classification contenant tous les emplois d'exécution, de maitrise jusqu'au cadre de collaboration. Par emploi de cadre de collaboration, il faut entendre celui exercé par le travailleur n'ayant pas le pouvoir de prendre à titre autonome des décisions de nature à influencer considérablement la marche de l'entreprise ${ }^{30}$.

A la lumière de l'article 90, sus évoqué, il est clairement établi que les cadres de direction ne sont pas concernés par l'application du SMIG. La dernière tension (10 ou 1000) correspond à la dernière classe de la catégorie de cadre de collaboration. Il appartient donc à chaque employeur, dans le cadre conventionnel, de fixer le salaire de cette catégorie des cadres non concernés ; il est évident qu'ayant franchi cette catégorie, leur traitement ne peuvent être ni égal, moins encore inférieur à celui alloué aux cadres de collaboration bénéficiaires de la tension 1000. Ainsi, toutes les fois qu'il serait question d'ajuster le salaire des travailleurs pour se conformer au SMIG actualisé, les cadres de direction ne seront pas concernés ; il faudra prévoir une autre négociation salariale qui tiendrait compte de leur statut.

2. Nationalité des travailleurs et son influence dans l'application du SMIG

Il est ici question d'interroger au sujet des travailleurs expatriés, de chercher à savoir si ceux-ci sont soumis au SMIG tel que fixé par l'autorité étatique en RD Congo ou par le salaire minimum applicable dans leurs pays respectifs.

Pour répondre à cette question, l'analyse part de l'article 86 , alinéa $1^{\text {er }}$ du code du travail qui dispose qu'à conditions égales de travail, de qualification professionnelle et de ren-

30 Article 90, alinéa 2 du code du travail de 2002. 
dement, le salaire est égal pour tous les travailleurs, quels que soient leur origine, leur sexe et leur âge.

Cette disposition légale justifie l'obligation de conformer la législation congolaise aux instruments juridiques internationaux qui proclament la non-discrimination et l'égalité de traitement en matière d'emploi ${ }^{31}$.

$\mathrm{Au}$ regard de cette disposition, la détermination du salaire au sein d'une entreprise doit en principe se faire en tenant compte des éléments objectifs tels la durée de prestation, la qualification professionnelle et le rendement. Ces conditions doivent s'observer sans tenir compte ni du sexe, ni de l'âge, ni de l'origine, notamment la nationalité, ni de la religion.

De ce fait, nous sommes d'avis que la nationalité du travailleur n'est pas opérant dans la détermination du salaire. Ainsi, c'est à bonne réflexion de soutenir que la pratique consistant à rémunérer différemment les salariés expatriés de même rang avec les mêmes qualifications professionnelles par rapport aux nationaux de même rang avec les mêmes qualifications professionnelles est contraire à la loi. Le motif lié au mode de vie des salariés expatriés justifiant la différence des salaires par rapport aux nationaux est discriminatoire et constitue une faute dans le chef de l'employeur ${ }^{32}$.

Outre cette disposition, il y a lieu de faire prévaloir également l'argument tiré du champ d'application du texte fixant le salaire minimum interprofessionnel garanti. En effet, il est élémentairement connu que le code du travail ainsi que toutes ses mesures d'exécution dont le texte qui fixe le SMIG s'appliquent à tous les travailleurs et à tous les employeurs, y compris ceux des entreprises publiques exerçant leur activité professionnelle sur l'étendue de la République Démocratique du Congo, quels que soient la race, le sexe, l'état-civil, la religion, l'opinion politique, l'ascendance nationale, l'origine sociale, la nationalité des parties, la nature des prestations, la rémunération ou le lieu de conclusion du contrat, dès lors que ce dernier s'exécute en République Démocratique du Congo ${ }^{33}$.

La fixation du SMIG qui est une mesure réglementaire prise en exécution de la disposition légale ne peut être discriminatoire et concerner uniquement les nationaux, de sorte qu'à l'égard des expatriés, il faille recourir au SMIG de leur pays d'origine pour déterminer leur salaire. Néanmoins, dans la négociation salariale avec un expatrié se trouvant à son pays au moment d'embauche, l'employeur le sollicite en ayant à l'esprit que son déplacement pour un autre pays nécessite la prise en compte de certains paramètres susceptibles de lui assurer un mode de vie plus ou moins égal à sa vie ordinaire dans son pays. C'est ce qui justifierait parfois la différence de salaire avec les nationaux, sans que cela soit fondé sur l'obligation de conformité au SMIG du pays dont il est ressortissant.

C'est donc à tort, de la part par exemple des inspecteurs de la DGI dans le cadre d'une mission de contrôle consistant à reconstituer l'assiette imposable de l'impôt exceptionnel sur la rémunération des expatriés, de se référer au SMIG applicable dans le pays dont le

31 Notamment les conventions $\mathrm{n}^{\circ} 100$ et 111 de l'OIT.

32 V. ILUNGA KASONGO, Code du travail, modifié, complété et annoté, Kinshasa, 2019, p. 96.

33 Article $1^{\text {er }}$ du code du travail de 2002. 
travailleur a la nationalité pour déterminer son salaire qui ne peut pas être inférieur à ce SMIG.

\section{Contrôle du respect par les employeurs du SMIG}

Le professeur MUKADI souligne que le législateur a instauré une série de mesures qui permettent de s'assurer du respect des dispositions en matière du SMIG. Il cite notamment l'obligation faite à l'employeur de remettre au travailleur au moment du paiement un décompte écrit de la rémunération payée (article 103 du code du travail), celle de mentionner, dans le contrat de travail écrit (...), le montant de la rémunération et des autres avantages (article 212 du code du travail), celle de tenir un livre de paie, lequel mentionne, pour chaque paie, toute somme due à titre de rémunération (article 213) (4. $^{34}$

La mission de contrôler le respect par les employeurs des obligations légalement imposées, tendant particulièrement au respect du SMIG, fait concourir plusieurs acteurs agissant à des degrés différents avec des pouvoirs également différents. Parmi ces acteurs, citons notamment les inspecteurs du travail, les contrôleurs de la DGI et de la CNSS à l'occasion de déclaration et paiement de l'impôt professionnel sur la rémunération, de l'impôt exceptionnel sur la rémunération des expatriés ainsi que des cotisations de la sécurité sociale. Outre ces acteurs extérieurs à l'entreprise, les syndicats des travailleurs sont également concernés dans la lutte pour le respect par les employeurs du SMIG fixé.

L'article 187 du code du travail dispose en effet que l'inspecteur du travail a pour mission de : assurer l'application des dispositions légales relatives aux conditions de travail et à la protection des travailleurs dans l'exercice de leur profession, telles que les dispositions relatives à la durée du travail, aux salaires, à la sécurité, à l'hygiène et au bien-être, à l'emploi des femmes, des enfants et des personnes avec handicap, ...

Vital ILUNGA note avec raison qu'on ne peut toujours pas faire confiance aux employeurs pour l'application des règles édictées par le présent code ou les décrets (ordonnances) présidentiels et actes réglementaires en la matière, d'où l'importance de l'inspection du travail qui est le complément nécessaire d'une réglementation du travail, et qui est destinée à assurer l'application des lois et règlements pour la protection généralement des travailleurs ${ }^{35}$.

Il est regrettable de constater l'inefficacité de l'action des inspecteurs du travail dans le cadre de la mission de veiller au respect par les employeurs du SMIG. Autant d'entreprises ne l'appliquent, encore qu'elles ne font aucune catégorisation professionnelle permettant de bien appliquer le SMIG. Au cours de l'exécution du contrat de travail, il est étonnant de remarquer qu'il existe moins des dossiers, à la limite rien du tout, sur des employeurs ne 
respectant pas le SMIG, alors qu'ils ne sont pas à rechercher ${ }^{36}$. Il est alors pertinent de réfléchir sur des problèmes pratiques qui constituent une pesanteur à l'application du SMIG.

\section{Problèmes pratiques de mise en œuvre du salaire minimum interprofessionnel garanti}

L'application du SMIG a toujours suscité des problèmes. D'un côté, les travailleurs tiennent à son strict respect alors que de l'autre côté, les employeurs posent des conditions, soit pour échelonner le paiement, soit pour obtenir des aménagements à l'application. Certains vont jusqu'à faire valoir la libre négociation salariale avec leurs travailleurs aux fins d'arrêter des salaires inférieurs au SMIG, sous prétexte de la taille financière de l'entreprise. Ce point examine tour à tour la question de la nature juridique du SMIG et les conséquences liées à son non-respect $(\mathrm{A})$, des problèmes de l'application du SMIG dans les entreprises à bas rendement $(\mathrm{B})$.

\section{Nature juridique du SMIG et ses conséquences en cas de non-respect}

La nature juridique du SMIG trouve son essence dans le fondement philosophique de ce dernier consistant, l'avons-nous dit, à assurer un équilibre en prévenant les abus des employeurs lors de détermination des salaires. Le respect du minimum légal a été érigé non seulement en obligation, mais également son non-respect est constitutif d'infraction conformément à l'article 321 du code du travail ${ }^{37}$.

Fort de ce qui précède, disons que la décision fixant le SMIG est d'ordre public.

Ce caractère d'ordre public ressort dans la plupart des législations. En France par exemple, tout salarié, quel que soit son statut sur le territoire français, a droit à une rémunération minimale au moins égale au Smic pour le nombre d'heures qu'il a effectuées, quel que soit le mode de rémunération pratiqué (même le travailleur à domicile à grande autonomie), et quelles que soient les stipulations de son contrat ${ }^{38}$. Ce même auteur cité écrit que si la loi a enfin permis aux accords d'entreprise de déroger aux conventions de branche, elle l'a expressément interdit en matière de salaires minima et de classifications. En ce domaine,

36 Les réclamations portant sur l'ajustement de salaire au SMIG sont à ressortir à l'occasion d'une plainte initiée consécutivement à la résiliation du contrat. Il est extrêmement rare de trouver une plainte, déposée à l'inspection du travail avant la résiliation du contrat, consistant à amener l'employeur à appliquer le SMIG.

37 Sont punis d'une amende qui ne dépasse pas 20.000 F.C. constants, les auteurs des infractions aux dispositions : a) des articles 6 literas a), b), c) et d), 8, 18, 19, 20, 21, 25, 26, 33 alinéa 2, 44, 47, 51,55 alinéa $3,56,60,64,65,66,78,79,84,89,90,98,99,100,101,103,111,112,114,116$, $117,118,119,121,122,125,126,129,133,136,137$ alinéa $2,138,140,141,142,143,144,145$, 146, 148 alinéa 1, 152, 154, 157, 167, 176, 178, 181, 212, 213, 215, 216, 217, 218, 221, 229, 234, $258,265,268,269 ;$ b) des décrets prévus aux articles 87 et $123 ; \ldots$.

38 Jean-Emmanuel Ray, Droit du travail. Droit vivant, 2014/2015, $23^{\text {ème }}$ édition, Paris, 2014, p. 169. 
la bonne vieille hiérarchie des normes conventionnelles demeure pour éviter le dumping social $^{39}$.

Les salaires minima interprofessionnels garantis sont, comme le note si bien Vital ILUNGA, intangibles et donc, d'ordre public. Ils sont insusceptibles d'être rabattu, même avec l'accord express du salarié, mais susceptible d'être augmenté par les parties au contrat ${ }^{40}$. Il est d'application immédiate dès lors qu'il entre en vigueur. Le juge ne peut nullement faire appliquer un SMIG abrogé même si le litige est né avant l'abrogation, même si la loi abrogée était favorable à la partie qui s'en prévaut. Il ne peut pas non plus appliquer un salaire conventionnel violant le $\mathrm{SMIG}^{41}$.

Le caractère d'ordre public qui détermine le SMIG constitue une véritable barrière protectrice des travailleurs contre le ravin dangereux d'infériorisation des salaires par les employeurs. Cependant, en pratique des problèmes se présentent, surtout à l'égard des employeurs dont le rendement mensuel n'est pas significatif pour couvrir la charge sociale à la hauteur du SMIG fixé.

\section{Application du SMIG par les employeurs à faible rendement}

Il est vrai que le respect du SMIG est un principe d'ordre public qui n'admet pas des compromis allant dans le sens de déterminer le salaire inférieur au minimum légal arrêté.

Cependant, parlant des modalités de la fixation du SMIG, le professeur MUKADI BONYI écrit que « sont pris en considération pour la fixation et l'ajustement du Smig les critères sociaux et économiques suivants. Dans les premiers, poursuit-il, il est tenu compte des besoins essentiels du travailleur, du coût de la vie et ses fluctuations, du niveau général de salaires, des prestations de la sécurité sociale, du niveau de vie comparé à celui d'autres groupes sociaux ; dans les seconds, il s'agit de la situation économique générale, la capacité de paiement des employeurs, la productivité, la classification des emplois, l'indice des prix, l'érosion monétaire $»^{42}$.

Parmi les critères ci-haut présentés qui concourent à la fixation du SMIG, certains concernent la vie du travailleur dont il faut garantir un certain standing alors que d'autres visent l'employeur qui subit le coût de la charge sociale sur laquelle le SMIG aura impacté.

39 Idem, p. 171.

40 V. ILUNGA, note 32, p. 98.

41 Dans l'affaire sous RT 722 du 14 juin 2012, opposant la Société Internationale CUSTOMS AGENCY Sprl et Monsieur TELAMA VA-KANDA Michel, le Tribunal de Grande Instance de Matadi avait décidé que « la dernière rémunération d'un salarié est son dernier salaire qui peut être soit conventionnel dans le respect du minimum prévu par la loi, soit légale lorsque celui conventionnel est en violation de la loi. Dans le cas d'espèce, le salaire conventionnel étant illégal, le tribunal condamne l'employeur au dernier salaire calculé selon le SMIG, en vigueur retenu par l'inspecteur du travail ». (Tiré de V. ILUNGA, note 32, p. 97).

42 MUKADI BONYI, note 34, pp. 236-237. 
Il est vrai qu'avant la fixation du SMIG, on procède à une concertation des partenaires sociaux. A l'occasion de cette concertation, les employeurs sont représentés en RDC par la Fédération des Entreprises du Congo (FEC) qui agit en tant que syndicat des employeurs. Par présomption, la présence de la FEC a comme conséquence l'adhésion des employeurs au SMIG à décider. C'est donc comme l'exemple du Plan qui, dans son élaboration et pour qu'il ait plus de chance de réussir, requiert la participation des acteurs en présence afin qu'il soit considéré comme une décision collective, un bien commun, ayant obtenu l'adhésion de tous.

Mais la question qui se pose est celle de s'interroger sur la représentativité de toutes les catégories d'employeurs au sein de la FEC. Il est en effet, une catégorie d'employeurs dont le rendement mensuel n'atteint parfois pas le chiffre qu'impose la masse salariale issue de l'application du SMIG alors que le besoin du maintien d'un tel effectif du personnel se fait sentir au sein de l'entreprise. Deux réalités se trouvent ainsi en affrontement; la première consistant à faire valoir le caractère d'ordre public du SMIG milite pour l'application ipso facto sans tenir compte de la taille de l'entreprise. La deuxième est celle qui tient compte de la capacité réelle de l'employeur à prendre en charge la masse salariale commandée par l'application du SMIG. La deuxième réflexion est très proche de la réalité dans les pays en développement où l'on peut constater la prépondérance des petites unités économiques, employant pourtant un nombre considérable des travailleurs dans une société à taux de chômage inimaginable. Malheureusement, aucune ouverture n'est faite légalement au sujet de l'application du SMIG, accordant la possibilité de négociation des salaires inférieurs au SMIG.

Au lieu d'amener les employeurs à procéder à la réduction des effectifs par le licenciement pour motif économique, solution juridique réconfortant la première tendance, nous pensons qu'il faille adapter le principe d'application du SMIG aux réalités pratiques de certaines catégories d'entreprises, en adoptant le système du SMIG progressif, dont les critères d'assujettissement des employeurs seront en même temps bien définis. C'est le cas notamment d'une structure hospitalière qui exige, pour son fonctionnement, la présence du personnel soignant, d'un travailleur commis au laboratoire, d'un administratif et des ouvriers pour la salubrité. Il faudrait dans ce cas, au moins cinq travailleurs pour faire fonctionner une telle structure dont parfois le rendement issu des services rendus n'atteint pas le SMIG de ces cinq travailleurs, même pris en tant que manœuvres ordinaires. En même temps que la structure médicale rend service à la société, au même moment, les cinq travailleurs échappent aux effets néfastes du chômage. Les pousser au licenciement au nom du respect du caractère d'ordre public du SMIG serait antisocial.

Cette position n'est cependant pas sans risque en ce qu'elle est susceptible d'occasionner une évasion salariale par les employeurs qui voudront se ranger vers des catégories d'employeurs favorisées. Face à ce risque, de lege ferenda, il faudrait instituer, préconiser le système d'admission des employeurs à telle ou telle autre catégorie. Ainsi, lors de l'examen du dossier par l'inspection du travail conjointement avec les agents du ministère de l'Economie, l'admission des employeurs peut objectivement être accordée. 


\section{Conclusion}

L'article 36, alinéa $1^{\text {er }}$ de la constitution de la RDC du 18 février 2016, telle que modifiée par la loi n¹1/002 du 20 janvier 2011 portant révision de certains articles de la Constitution de la République Démocratique du Congo du 18 février 2006, pose le principe selon lequel le travail est un droit et un devoir sacrés pour chaque Congolais. Le travail dont question ici est celui qui met en relation une personne physique, appelée travailleur, et une autre personne, physique ou morale, appelée employeur, les deux ayant conclu un contrat de travail, défini comme toute convention, écrite ou verbale, par laquelle la première s'engage à fournir à la deuxième un travail manuel ou autre sous sa direction et son autorité moyennant rémunération.

Que référence soit faite à la législation congolaise, à la convention $n^{\circ} 100$ de l'Organisation Internationale du Travail (OIT) ou à toute autre législation, il est admis que rémunération et salaire se diffèrent. Le salaire a toujours été présenté comme prix du travail. C'est donc le salaire qu'il faille mesurer à la prestation fournie par le travailleur au profit de l'employeur. Il revêt un caractère sacré dans la mesure où il constitue la récompense de la sueur perdue par le travailleur à l'occasion de l'exécution de son contrat.

Cependant sa détermination, laissée en principe à la liberté des parties, fait l'objet de beaucoup d'attention ; pas seulement en raison de son caractère sacré mais également compte tenu du risque encouru si tant est que les rapports professionnels qui en constituent l'essence sont par nature déséquilibrés. L'employeur, dont la position dominante n'est plus à démontrer, n'a généralement pas d'intérêt, dans un contexte de libre court, de prévoir un salaire décent au travailleur. La tendance est au contraire vers sa minimisation pour réduire sensiblement les charges au sein de l'entreprise. Cette situation se justifie par des principes du monde capitaliste où prédominent les intérêts du patron au détriment de ceux des ouvriers (travailleurs). L'interventionnisme étatique qui constitue la pesanteur de la liberté directrice du monde capitaliste se manifeste sous diverses formes, selon le domaine d'intervention.

En matière du travail, domaine plus exposé aux méfaits de la liberté capitaliste, l'Etat a développé beaucoup de mécanismes normatifs comme institutionnels en vue de garantir aux travailleurs la jouissance d'un certain nombre des droits, qualifiés de fondamentaux, parmi lesquels la décence de salaire.

A la suite des autres Etats, la RDC reconnait la liberté de fixation de salaire aux parties, soit à l'occasion des contrats individuels, soit par voie de conventions collectives. Mais, elle place une importante barrière en fixant un montant minimum en deçà duquel les parties ne peuvent pas déterminer le salaire (le législateur parle de la rémunération). Il s'agit donc du salaire minimum interprofessionnel garanti (SMIG), dont la compétence est légalement attribuée au président de la République. Il a été démontré que l'actuel décret (du premier ministre) fixant le SMIG à 7.075CDF n'est pas conforme à la législation et son application aurait dû poser problème en qu'il ne pourrait pas juridiquement abroger l'ordonnance $n$ 
๑80/040 de 2008 fixant le SMIG à 1.680Fc, le décret étant une norme inférieure à l'ordonnance. Mais il faut avant tout obtenir son annulation en ce qu'il est illégal.

Cette étude a eu l'occasion de répondre à certaines questions en rapport avec le SMIG, à savoir ses champs d'application (matériel, territorial et personnel), sa nature juridique et les conséquences de son inapplication, ainsi que des problèmes pratiques que suscite son application particulièrement concernant les employeurs à faible rendement financier.

Ayant le caractère d'ordre public non-susceptible d'accommodement entre employeur et travailleur, le SMIG devait à notre point de vue se comparer au salaire de base, contrairement à la confusion volontairement semée par le législateur congolais, et doit s'appliquer sans discrimination à tous les travailleurs nationaux et expatriés se trouvant sur l'étendue du territoire congolais. Au vu de son caractère d'ordre public, le non-respect du SMIG a été érigé en infraction ${ }^{43}$, mettant ainsi tous les employeurs sur le même diapason, y compris ceux à faible rendement employant néanmoins une franche importante des travailleurs. Pour tenir compte de ces petites entreprises dont l'apport n'est pas moins considérable, dans une société de chômage, nous avons proposé la catégorisation des employeurs et l'institution de Smig progressif, avec un mécanisme de contrôle avant comme après l'admission à telle ou telle catégorie. Cette dernière idée proposée fait suite à beaucoup de discussions engagées avec ces catégories d'employeurs, à l'occasion de la mise en application, malheureusement, du SMIG de 2018 fixant le salaire du manœuvre ordinaire à 7075CDF.

\section{REFERENCES BIBLIOGRAPHIQUES}

BAVOZET Franck, Social 2016, Paris, 2016.

CARMELYNCK G.H et LYON-CAEN, Droit du travail, Paris, 1965.

DIUMI SHUTSHA D., Six leçons de droit du travail, Kinshasa-Bruxelles, 2018.

$F E C$, Département Juridique, Social et Fiscal, « le salaire minimum interprofessionnel garanti en application en République Démocratique du Congo ", in Veillé juridique en entreprise, n007/DJSF/ 2018, novembre 2018.

ILUNGA KASONGO V., Code du travail, modifié, complété et annoté, Kinshasa, 2019.

KUMBU ki NGIMBI Jean-Michel, Droit social, Kinshasa, 2018.

MASANGA PHOBA MVIOKI, Droit du travail, Kinshasa, 2018

MAZYAMBO MAKENGO KISASA A., Régionalisme et décentralisation en République Démocratique du Congo, in La consolidation du cadre démocratique en République démocratique du Congo, modules de renforcement des capacités à l'intention des institutions parlementaires, PNUD, Kinshasa

MUKADI BONYI, Droit du travail, Kinshasa, 2008.

OIT, Conventions et recommandations internationales du travail 1914-1984, volume I, BIT, Genève, 1985.

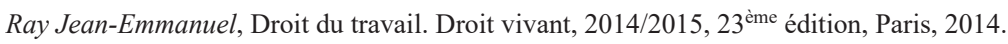

